\title{
26 Rektumkarzinom
}

Julia L. S. Hardt, Stefan Post

\subsection{Kapitelzusammenfassung}

Die MRT-Untersuchung des Beckens stellt den Goldstandard beim lokalen Staging des Rektumkarzinoms dar. Ergänzend zum Einsatz kommen die rektale Endosonographie und die starre Rektoskopie. Die Therapie des Rektumkarzinoms ist multimodal aufgebaut und setzt sich aus den einzelnen Modalitäten Radiotherapie, Radiochemotherapie, Chemotherapie und Operation zusammen. Die Zusammensetzung der Therapie hängt von der Tumorlokalisation sowie dem Tumorstadium ab. Bei gut oder mäßig differenzierten pT1-Karzinomen ohne Lymphgefäßinvasion (low-risk Histologie), ist eine lokale Tumorexzision mittels endoskopisch-mikrochirurgischer Resektion als alleinige kurative Therapie onkologisch ausreichend, sofern eine R0-Resektion erzielt werden kann. Als Methoden kommen die transanale endoskopisch-mikrochirurgische Resektion (TEM) und transanale endoskopische Operation (TEO) infrage. Den Goldstandard der radikalchirurgischen Therapie des Rektumkarzinoms stellt die total mesorektale Exzision (TME) dar, welche die Lokalrezidivrate erheblich senkt. Im Kontext der radikalchirurgischen Therapie sind nach deutscher S3-Leitlinie die (tiefe) anteriore Rektumresektion, die abdomino-perineale Rektumexstirpation und die intersphinktäre Rektumresektion als onkologisch gleichwertig anzusehen. Für alle drei OP-Verfahren gelten die gleichen onkologischen Grundprinzipien. Die Rekonstruktion der Darmkontinuität sollte nach sphinktererhaltenden Resektionen durch eine Seit-zu-End Anastomose oder einen Kolon-J-Pouch erfolgen. Bei sehr ausgedehnten perinealen Defekten nach abdomino-perinealer Rektumexstirpation sollte die Rekonstruktion mittels myokutanem Lappen in Erwägung gezogen werden, beispielsweise unter Verwendung des Musculus rectus abdominis oder des Musculus glutaeus maximus. Die laparoskopische Rektumresektion ist nach aktuellem Evidenzstand bei entsprechender Expertise des Operateurs eine onkologisch gleichwertige Alternative zur offenen Chirurgie. Unter den intraoperativen Komplikationen zählen Verletzungen von Ureter, Blase und Vagina sowie Blutungskomplikationen zu den relevantesten, postoperativ tragen Nachblutungen, Wundinfekte, Anastomoseninsuffizienz, Blasenentleerungsstörungen und Stomakomplikationen zur Morbidität bei. Um die aus der Anastomoseninsuffizienz resultierende Morbidität und Mortalität zu senken, sollte leitliniengerecht im Rahmen einer tiefen anterioren Rektumresektion mit tiefer Anastomose ein Deviationsstoma angelegt werden. Die onkologische Prognose hängt von Patienten-, Tumor- und Therapie-assoziierten Faktoren ab, wobei die Radikalität und Qualität der chirurgischen Resektion eine entscheidende Rolle spielen.

Hinweis: Zu Inzidenz, Epidemiologie, Definition, TNM-Klassifikation, Symptomatik, Differentialdiagnosen, allgemeiner Diagnostik und Nachsorge siehe Kap. 25 
Kolonkarzinom. In diesem Kapitel werden nur die darüberhinausgehenden Spezifika des Rektumkarzinoms abgehandelt.

\subsection{Spezielle Diagnostik des Rektumkarzinoms}

\subsubsection{MRT}

Während zur Metastasensuche bei weiterem Abklärungsbedarf nach Röntgen-Thorax und Abdomen-Sonographie die Computertomografie (CT) Einsatz findet, gilt die Magnetresonanztomografie (MRT) als Goldstandard beim lokalen Staging. MR-morphologisch kommen der topografische Bezug des Tumors zur Grenzlamelle des Mesorektums sowie zu den Nachbarorganen im kleinen Becken zur Darstellung. Einen besonderen Vorteil bietet das MRT durch die akkurate Abbildung der mesorektalen Faszie und des zirkumferentiellen Resektionsrandes (engl. circumferential resection margin, CRM), siehe Abb. 26.1 und 26.2. In der Literatur ist gut belegt, dass die MRTBildgebung einen hohen prädiktiven Wert hinsichtlich des Befalls des CRM hat: ist der CRM MR-morphologisch negativ d.h. $>1 \mathrm{~mm}$, so wird er dies mit über $90 \%$ iger Wahrscheinlichkeit auch am resezierten Präparat, also in der Befundung durch den Pathologen, sein. Laut einer Metaanalyse von 21 Studien erreicht die MRT eine Sensitivität von $77 \%$ und eine Spezifität von $94 \%$ in Bezug auf die Vorhersagegenauigkeit des CRM-Status. Das Rektumkarzinom stellt sich üblicherweise hypointens in Abgrenzung zur normalen Darmwand dar. Bei Infiltration des externen Sphinkters liegt eine T3-Situation vor, bei Infiltration des M. levator ani bereits eine T4-Kategorie. Eine T4-Kategorie ist ebenfalls zu befunden, wenn der Tumor die mesorektale Faszie überschreitet und das viszerale Peritoneum infiltriert (T4a) oder gar Nachbarorgane (T4b) wie beispielsweise Uterus, Harnblase, Prostata oder Samenbläschen. Die Vorhersagegenauigkeit der MRT bezogen auf die T-Kategorie ist hoch: laut der bereits oben zitierten Metaanalyse liegt die Sensitivität bei $87 \%$, die Spezifität bei $75 \%$. Des Weiteren wird der Lymphknotenstatus beurteilt, der einen unabhängigen Prädiktor des Gesamtüberlebens darstellt. Als MR-morphologische Kriterien für einen Tumorbefall eines Lymphknotens gelten Größe $>5 \mathrm{~mm}$, irreguläre Kontur und inhomogene Signalintensität, siehe Abb. 26.2; allerdings ist die sichere Detektion von Lymphknotenmetastasen schwierig. Neben der Abklärung von TN(M)- und CRM-Status dient das MRT aber auch der Beurteilung der extramuralen venösen Infiltration (EMVI). Diese liegt vor, wenn der Tumor große Gefäße, meist Venen, in der Nähe der Muscularis propria infiltriert und stellt einen unabhängigen Prädiktor der onkologischen Prognose dar. Gemeinsam mit der rektalen Endosonographie nimmt die MRT einen entscheidenden Stellenwert im Rahmen der präoperativen Diagnostik ein. In Abhängigkeit von den erhobenen Befunden wird über die Indikation für eine neoadjuvante Therapie (bei Infiltration ins Mesorektum, T3-Kategorie) entschieden und das operative Vorgehen geplant. Das lokale Staging ist beim Rektumkarzinom von großer Bedeutung, da bei- 
spielsweise ein low-risk T1-Karzinom lokal abgetragen werden kann, ein high-risk T1oder ein T2-Karzinom hingegen einer onkologisch radikalen Resektion bedürfen.
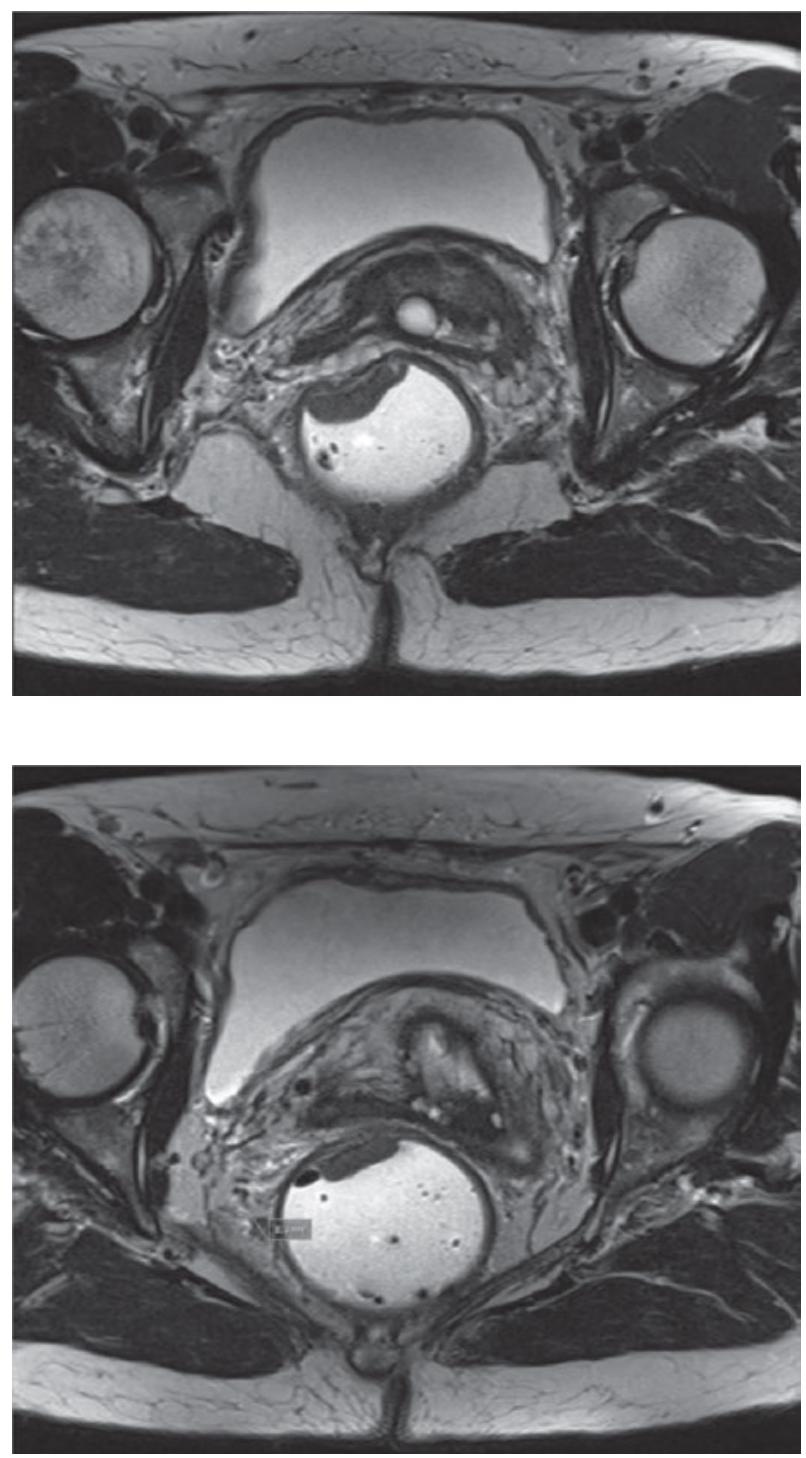

Abb. 26.1: Staging-MRT (prätherapeutisch) - Rektumkarzinom $5 \mathrm{~cm}$ ab ano bei $9-12 \mathrm{Uhr}$ SSL, überwiegend auf die Rektumwand beschränkt, anterolateral jedoch V. a. $2 \mathrm{~mm}$ tiefe Infiltration des mesorektalen Fetts, somit MR-morphologisch einer T3b-Kategorie entsprechend.

Abb. 26.2: Staging-MRT derselben Patientin mit tiefsitzendem Rektumkarzinom und metastasensuspektem mesorektalem Lymphknoten rechts mit irregulärer, lobulierter Berandung und einem Durchmesser $>5 \mathrm{~mm}$, somit MR-morphologisch V. a. N1 Kategorie. 


\subsubsection{Endosonographie}

Die rektale Endosonographie (REUS) stellt eine wichtige zur MRT-Diagnostik komplementäre Modalität im Rahmen des lokalen Stagings dar. Insbesondere bei frühen T-Stadien erweist sich die REUS als vorteilhaft: bei der Abgrenzung beispielsweise von T1- versus T2-Kategorie besitzt sie bei gleich hoher Sensitivität wie das MRT eine bessere Spezifität. Deshalb empfiehlt die aktuelle deutsche S3-Leitlinie auch, dass bei Verdacht auf ein T1-Karzinom eine Endosonographie durchgeführt werden soll, zum lokalen Staging fortgeschrittener Stadien hingegen eine MRT. Bei großen, stenosierend und/oder im proximalen Rektum wachsenden Karzinomen stößt die REUS an ihre Grenzen. Die Beurteilung des N-Status ist ebenso wie im Rahmen der MRT fehleranfällig bzw. ungenau. Als Malignitäts-suspekt gelten in der REUS Lymphknoten, die größer $5 \mathrm{~mm}$, irregulär berandet und echoarm sind. Die mesorektale Faszie lässt sich in der REUS nicht darstellen. Eine weitere Limitation der REUS stellt auch ihre Untersucherabhängigkeit dar; dennoch hat sie neben der MRT ihren festen Stellenwert beim lokalen Staging des Rektumkarzinoms.

\subsubsection{Starre Rektoskopie}

Auch wenn die starre Rektoskopie mit Bestimmung des Tumorunterrandes und seiner Entfernung von der Linea dentata laut deutscher S3-Leitlinie obligat im Rahmen der präoperativen Diagnostik durchzuführen ist, ist bei entsprechender Expertise (Messung am gestreckten Gerät im Rückzug) die flexible Endoskopie vergleichbar genau. Bezüglich der Beurteilung der Höhe des Tumorunterrandes ist die Endoskopie der MRT weiterhin überlegen, wohingegen die MRT zur Bestimmung der Tumorhöhe in Relation zur peritonealen Umschlagfalte notwendig ist, da diese endoskopisch nicht bestimmbar ist.

\subsection{Multimodale Therapiekonzepte beim nicht-metastasierten Rektumkarzinom}

Die Therapie des Rektumkarzinoms hat in den letzten Jahrzehnten relevante Fortschritte gezeigt und ist zunehmend multimodal und interdisziplinär aufgebaut. Die multimodale Therapie setzt sich dabei aus den einzelnen Modalitäten Radiotherapie, Radiochemotherapie, Chemotherapie und Operation zusammen. Laut aktueller deutscher S3-Leitlinie und vor dem Hintergrund sehr niedriger Lokal- und Fernmetastasierungsraten nach alleiniger onkologischer Rektumresektion werden cT1/2NO-Tumoren (UICC Stadium I) des mittleren und unteren Rektumdrittels sowie Rektumkarzinome des oberen Drittels ohne Risikokonstellation für ein Lokalrezidiv oder eine R1-Situation nicht neoadjuvant vorbehandelt. Rektumkarzinome im oberen Drittel werden in 
der Regel analog zum Kolonkarzinom therapiert. Demgegenüber ist in der Regel bei lokal fortgeschrittenen Tumoren (cT3/4 und/oder N+) des mittleren und unteren Rektumdrittels die neoadjuvante Radiochemotherapie indiziert. Vor dem Hintergrund, dass Karzinome der Kategorie T3a,b bei erwartet sicher negativem CRM eine vergleichbare Lokalrezidivrate wie Karzinome der Kategorie T2 haben, kann in diesen Fällen und bei negativer N-Kategorie auf eine neoadjuvante Radiatio verzichtet werden. Alternativ zur einer neoadjuvanten Radiochemotherapie kann auch eine KurzzeitRadiatio $(5 \times 5 \mathrm{~Gy})$ erfolgen, was seit Publikation der niederländischen TME-Studie 2001 auf solider Evidenz basiert. Die Lokalrezidivrate wurde in dieser Studie durch die Kurzzeitbestrahlung gegenüber der alleinigen TME signifikant reduziert. Wissenschaftlich als Standard etabliert wurde die neoadjuvante Radiochemotherapie durch die deutsche CAO/ARO/AIO-94 RCT, eine Phase III-Studie, die an 823 Patienten mit Rektumkarzinom der UICC-Stadien II und III die neoadjuvante (präoperative) Radiochemotherapie mit 50,4 Gy und 5-Fluorouracil mit der adjuvanten (postoperativen) verglich. Die kumulative 5-Jahres-Inzidenz an Lokalrezidiven betrug im neoadjuvanten Arm $6 \%$ versus $13 \%$ im adjuvanten, was einem signifikanten Unterschied entsprach. Das 5-Jahres-Gesamtüberleben hingegen unterschied sich nicht signifikant und lag bei 76\% (neoadjuvanter Arm) bzw. $74 \%$ (adjuvanter Arm). Die von Sauer et al. nach einem medianen Follow-up von 11 Jahren publizierten Langzeitergebnisse bestätigten dies: auch nach 10 Jahren fand sich noch eine signifikante Verbesserung der lokalen Kontrolle, allerdings ergab sich weiterhin kein Unterschied zwischen den beiden Therapiearmen hinsichtlich des Gesamtüberlebens.

Nach der onkologischen Rektumresektion ist in Abhängigkeit vom Tumorstadium eine adjuvante Chemotherapie indiziert: im UICC Stadium I (pT1/2NO) und bei R0-Resektion besteht hierfür keine Indikation. Dagegen sollte bei Vorliegen histopathologisch nachgewiesener Risikofaktoren für ein Lokalrezidiv im Regelfall eine adjuvante Therapie appliziert werden, sofern nicht bereits präoperativ bestrahlt wurde, als Radiochemotherapie. Ob in dieser Situation eine postoperative Bestrahlung auch bei guter TME-Qualität erfolgen sollte, ist umstritten. Eine tabellarische Übersicht über die stadiengerechte Therapie des Rektumkarzinoms bietet Tab. 26.1.

Viel diskutiert in der wissenschaftlichen Literatur und in Fachkreisen wurde in den letzten Jahren die Frage nach dem abzuwartenden Intervall nach Abschluss der neoadjuvanten Radiochemotherapie. Nach deutscher Leitlinie sollte nach erfolgter Kurzzeit-Radiatio ( $5 \times 5$ Gy) die Operation innerhalb von 10 Tagen nach Beginn dieser oder erst nach 4-8 Wochen durchgeführt werden. Letzteres stützt sich auf die Ergebnisse der Stockholm III-Studie mit 545 randomisierten Patienten, die entweder eine Kurzzeitbestrahlung mit direkt anschließender Operation, einer Kurzzeitbestrahlung mit Operation nach 4-8 Wochen oder eine Langzeitbestrahlung mit Operation nach 4-8 Wochen erhielten: Patienten mit Kurzzeitbestrahlung und Operation nach 4-8 Wochen wiesen signifikant frühere ypT-Stadien sowie eine signifikant höhere Rate an pCR auf. Für eine unmittelbar nach Kurzzeitbestrahlung zu planende Resektion sprechen dagegen die Daten aus einer Sekundäranalyse der niederländischen 
TME-Studie: insbesondere bei älteren Patienten ( $\geq 75$ Jahre) war die nicht durch die Krebserkrankung verursachte Mortalität signifikant höher, wenn zwischen dem Ende der Kurzzeitbestrahlung und der Operation mehr als 3 Tage lagen. Daher plädieren van den Broek et al. für einen maximalen Zeitraum von Kurzzeitbestrahlung und OP von 10 Tagen.

Auch bezüglich des optimalen Intervalls zwischen abgeschlossener neoadjuvanter konventionell fraktionierter Radiotherapie und Operation ist die Evidenzlage kontrovers:

Die multizentrische GRECCAR-6-RCT, in der 265 Patienten nach neoadjuvanter Radiochemotherapie entweder nach 7 oder 11 Wochen operiert wurden, ergab keinen signifikanten Unterschied hinsichtlich des primären Endpunkts pathologische Komplettremission (pCR, ypTONO), allerdings eine signifikant erhöhte Morbidität aufgrund internistischer Komplikationen sowie eine signifikant schlechtere TMEQualität im Arm mit langem Intervall, was darauf hindeuten könnte, dass die spätere Resektion technisch anspruchsvoller ist. Dagegen fand sich in zwei Metaanalysen nicht randomisierter Studien eine höhere Rate an Komplettremissionen bei nicht erhöhter Komplikationsrate. Da keine hochrangige Evidenz vorliegt, die einen Benefit durch ein längeres Intervall belegt, empfiehlt die deutsche Leitlinie weiterhin ein Intervall von 6-8 Wochen.

Wenn im Rahmen des Re-Stagings nach neoadjuvanter Therapie sowohl klinisch als auch endoskopisch und durch bildgebende Verfahren (MRT, REUS) kein Tumor mehr nachweisbar ist, kann leitliniengerecht auch gänzlich auf eine Operation verzichtet werden. Allerdings müssen diese Patienten für mindestens fünf Jahre (wahrscheinlich sogar länger) sehr engmaschig nachgesorgt werden. Die onkologische Sicherheit dieses Vorgehens ist bisher nicht mittels randomisierter Studien belegt. Eine 2017 veröffentlichte Metaanalyse von fünf retro- und vier prospektiven Beobachtungsstudien untersuchte die Rate an Tumorrezidiven, assoziierter „Salvage“-Chirurgie und onkologischem Outcome: von 370 Patienten in der „watch and wait“-Gruppe zeigten 256 (69\%) ein persistierendes komplettes Ansprechen nach neoadjuvanter Therapie. In den Fällen, in denen der Tumor wiederkehrte, war eine „Salvage“-Operation zu $84 \%$ möglich. Differenzen hinsichtlich Gesamtüberleben und krankheitsfreiem Überleben ergaben sich nicht zwischen der initial schon operierten und der „watch and wait“-Gruppe. Angesichts der limitierten Datenlage schlussfolgern die Autoren, dass das „watch and wait“-Konzept aktuell noch nicht als einer der Standards gelten kann. 
Tab. 26.1: Stadiengerechte Therapie des Rektumkarzinoms.

\begin{tabular}{|c|c|c|c|c|}
\hline $\begin{array}{l}\text { UICC- } \\
\text { Stadium }\end{array}$ & (c)TNM-Formel & $\begin{array}{l}\text { Neoadjuvante Radio-/ } \\
\text { Radiochemotherapie }\end{array}$ & Resektion/ OP & $\begin{array}{l}\text { Adjuvante } \\
\text { Chemotherapie }\end{array}$ \\
\hline 0 & TisNOMO & nein & lokale Exzision & nein \\
\hline I & T1/T2NOM0 & nein & $\begin{array}{l}\text { T1 low risk: } \\
\text { lokale Exzision } \\
\text { T1 high risk/ T2: } \\
\text { TME/PME }\end{array}$ & $\begin{array}{l}\text { Nein, falls prätherapeu- } \\
\text { tisches Staging korrekt }\end{array}$ \\
\hline $\begin{array}{l}\text { IIA } \\
\text { IIB }\end{array}$ & $\begin{array}{l}\text { T3NOMO } \\
\text { T4NOMO }\end{array}$ & $\begin{array}{l}\text { ja, wenn Tumorlokalisati- } \\
\text { on }<12 \mathrm{~cm} \text { ab ano }\end{array}$ & TME/ PME & $\begin{array}{l}\text { ja, unabhängig von } \\
\text { Tumorlokalisation }\end{array}$ \\
\hline $\begin{array}{l}\text { IIIA } \\
\text { IIIB } \\
\text { IIIC }\end{array}$ & $\begin{array}{l}\text { T1-2N1M0 } \\
\text { T3-4N1M0 } \\
\text { alle T, N2M0 }\end{array}$ & $\begin{array}{l}\text { (ja) } \\
\text { ja, wenn Tumorlokalisati- } \\
\text { on }<12 \mathrm{~cm} \text { ab ano }\end{array}$ & $\begin{array}{l}\text { TME/ PME } \\
\text { TME/ PME }\end{array}$ & $\begin{array}{l}\text { ja, wenn pT3/ } 4 \text { oder } \mathrm{N}+ \\
\text { ja, unabhängig von } \\
\text { Tumorlokalisation }\end{array}$ \\
\hline IV & alle $\mathrm{T}$, alle $\mathrm{N}, \mathrm{M} 1$ & $\begin{array}{l}\text { je nach Resektabilität der } \\
\text { Metastasen primäre Re- } \\
\text { sektion und anschließend } \\
\text { medikamentöse Tumor- } \\
\text { therapie oder zunächst } \\
\text { systemische Therapie und } \\
\text { ggf. sekundäre Resektion }\end{array}$ & & \\
\hline
\end{tabular}

\subsection{Operative Therapie des Rektumkarzinoms}

\subsubsection{Endoskopisch-mikrochirurgische Tumorresektion}

Gemäß S3-Leitlinie ist eine lokale Tumorexzision (Vollwandexzision) als alleinige kurative Therapie onkologisch ausreichend bei gut oder mäßig differenzierten pT1Karzinom $<3 \mathrm{~cm}$ und ohne Lymphgefäßinvasion (low-risk Histologie), sofern eine R0Resektion erzielt werden konnte. Auch wenn dies nicht Eingang in die Leitlinie fand, sind tiefe Submucosa-Infiltrationen (T1sm3) wohl eher als zu high-risk klassifizieren. An ihre Grenzen stoßen alle transanalen Techniken auf Höhe der peritonealen Umschlagfalte, da oberhalb dieser unklar ist, inwieweit die Resektion onkologisch ausreichend ist.

Neben der konventionellen, offenen lokalen Exzision nach Parks stehen heute verschiedene endoskopisch-mikrochirurgische Techniken zur Verfügung:

\section{Transanale endoskopisch-mikrochirurgische Resektion (TEM)}

Mittels TEM sind-im Gegensatz zur konventionellen Technik-auch Tumoren des oberen Rektums lokal exzidierbar. Entwickelt wurde die TEM in den 1980er Jahren 
von Gerhard Bueß, was einem Meilenstein in der Geschichte der transanalen Tumorchirurgie entspricht. Eine Metaanalyse nicht randomisierter Studien, welche die TEM mit der konventionellen transanalen lokalen Exzision hinsichtlich postoperativer Morbidität und onkologischer Resektat-Qualität verglich, fand bei vergleichbarer postoperativer Komplikationsrate eine höhere Rate negativer Resektionsränder sowie eine Reduktion der Raten an fragmentierten Resektaten und Lokalrezidiven nach TEM. Die bisher vorliegende limitierte Evidenz legt somit nahe, dass die TEM der konventionellen Technik bezüglich der onkologischen Qualität überlegen ist.

\section{Transanale end oskopische Operation (TEO)}

Die TEO entspricht einer modifizierten Variante der TEM, die im Vergleich zu dieser einen geringeren Materialaufwand mit sich bringt und zudem die Verwendung herkömmlicher endoskopischer Instrumente erlaubt. Allerdings bietet die TEM eine 3D-Optik, wohingegen das TEO-System nur eine 2D-Optik beinhaltet. Ansonsten sind TEO und TEM prinzipiell sehr ähnlich: beide Techniken bzw. Systeme arbeiten mit starren Plattformen (TEM; Richard Wolf GmbH, Knittlingen, Deutschland und TEO; Karl Storz GmbH \& Co. KG, Tuttlingen, Deutschland), sind wiederverwendbar, werden mittels Haltevorrichtung am OP-Tisch fixiert und mit beiden Methoden lassen sich auch Tumoren im oberen Rektumdrittel resezieren im Gegensatz zur konventionellen transanalen Resektion nach Parks. Insgesamt finden sich in der Literatur für TEM und auch TEO geringe Komplikationsraten, adäquate und der konventionellen transanalen Resektion überlegene onkologische und exzellente funktionelle Ergebnisse. Eine kleine RCT mit 36 Patienten, die die Ergebnisse nach TEM und TEO verglich, fand keine signifikanten Unterschiede hinsichtlich postoperativer Morbidität und Mortalität sowie in Bezug auf die Resektat-Qualität.

\section{Weitere minimalinvasive transanale Verfahren}

Unter den Begriffen „transanal minimal invasive surgery“ (TAMIS) und „transanal endoscopic surgery“ (TES) werden weitere minimal-invasive endoskopische Techniken zur Exzision von Rektumtumoren via Single-Ports subsummiert, u. a. SSL (Single-site laparoscopic access system, Ethicon Endo-Surgery, Cincinnati, OH, USA), GelPOINT Path Transanal Access Platform (Applied Medical, Rancho Santa Margarita, CA, USA) und SILS Port (Covidien-Medtronic, Minneapolis, MN, USA). Nachteil dieser flexiblen Systeme ist, dass der Port nicht mittels Haltearm fixiert wird, sondern anal in Position gehalten werden muss und daher bei sehr tiefsitzenden Befunden nachteilig sein kann, weil der Port nicht bis zur Linea dentata zurückzuziehen ist, ohne eine Dislokation zu riskieren. Bei allen Systemen wird nach Einbringen des analen Ports ein Kapnorektum erzeugt, um anschließend unter Einsatz einer $30^{\circ}$-Optik und mit Standard-Laparoskopie-Instrumenten operieren zu können.

Einen neuen, noch wenig mit Evidenz untermauerten Ansatz stellt der Einsatz von Roboter-assistierten TEM-Techniken dar: in kleinen Fallserien konnte die Mach- 
barkeit einer Da Vinci (Intuitive Surgical Inc, Sunnyvale, CA, USA) Si-assistierten TEM-Operation gezeigt werden. Für die Roboter-Assistenz sprechen u. a. die Elimination des Chirurgen-Tremors sowie die 7 Freiheitsgrade des Roboterarmes, die insbesondere auf engem Raum Vorteile bieten; dagegen sprechen die verlängerte OPDauer sowie der materielle und finanzielle Mehraufwand.

Die sogenannte TaTME (transanale TME) wurde mit dem Ziel entwickelt, auch in besonders schwieriger Situation (adipöser männlicher Patient mit engem Becken, voluminösem Mesorektum und tiefsitzendem Tumor) eine hohe TME-Qualität zu erreichen. In der Regel wird die TaTME als Hybrid-Eingriff durchgeführt, wobei der abdominelle Teil der Operation offen, laparoskopisch oder robotisch-assistiert ablaufen kann. Sofern die erforderlichen personellen Ressourcen verfügbar sind, können abdomineller und transanaler Part synchron erfolgen, was die OP-Zeit verkürzt und die Kooperation der beiden Teams mit verschiedenen Perspektiven bei sehr schwieriger mesorektaler Dissektion ermöglicht. Eine Auswertung der ersten 720 TaTME-Fälle aus dem internationalen TaTME-Register ergab eine überwiegend gute TME-Qualität (85\%: gut, $11 \%$ : moderat, 4\%: schlecht) und eine R1-Resektionsrate von nur 2,7\% bei ebenfalls akzeptabler Komplikationsrate (Morbidität 32,6\%, Mortalität 0,5\%). Da sie andererseits nicht ohne spezielle Risiken ist, muss die TaTME derzeit noch als experimentelle Methode angesehen werden, deren Stellenwert erst durch noch laufende multizentrische RCTs festgelegt werden wird.

\subsubsection{Total mesorektale Exzision (TME)}

Den Goldstandard der radikalchirurgischen Therapie des Rektumkarzinoms stellt seit Anfang der 1980er Jahre die von Bill Heald erstbeschriebene TME dar. Wie Heald 1988 konkretisierte, gilt es die Hüllfaszie des Mesorektums als sog. „holy plane“ bei der Präparation intakt zu belassen. Zahlreiche Studien haben seitdem gezeigt, dass die TME die Lokalrezidivrate erheblich senkt.

Entscheidend bei der Durchführung der TME ist die streng schichtgerechte Präparation (siehe Abb. 26.3) und dies nicht nur in onkologischer Hinsicht, sondern auch um das Risiko einer Schädigung der pelvinen Nervenplexus sowie einer Blutungskomplikation zur minimieren. Dorsalseitig erfolgt die Präparation bis zum Beckenboden im Spatium retrorectale, dem Raum zwischen der Fascia pelvis parietalis (Waldeyer-Faszie) und der mesorektalen Faszie. Nach beidseitiger, ventralseitiger Inzision des Peritoneums knapp oberhalb der peritonealen Umschlagfalte führt die ventrale Dissektion durch das Spatium praerectale, das ventral durch das vordere Blatt der Denonvilliers'schen Faszie und dorsal vom hinteren Blatt selbiger Faszie bzw. der vorderen Grenzlamelle des Mesorektums begrenzt wird. Bei der ventralen Dissektion muss ein besonderes Augenmerk darauf gerichtet werden, dass Vagina oder Samenbläschen nicht verletzt werden. Lateralseitig sind häufig schwieriger darzustellen, weshalb hier besonders vorsichtig präpariert werden muss. Das Rektum wird nach 


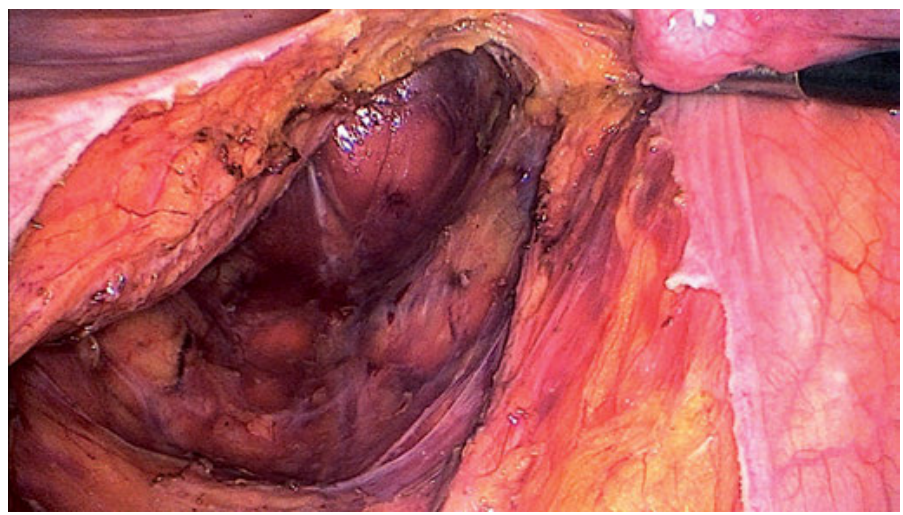

Abb. 26.3: Laparoskopische TME mit Darstellung der schichtgerechten Präparation, dorsal ist das Rektum weitgehend ausgelöst.

abgeschlossener TME auf Levatorenhöhe und somit unterhalb des Mesorektums abgesetzt.

Die PME (partielle Mesorektum Exzision) erfolgt analog zur TME, allerdings aboralwärts nicht bis auf Levatorenhöhe, sondern nur bis $5 \mathrm{~cm}$ distal des aboralen Tumorunterrandes.

Im Kontext der radikalchirurgischen Therapie des Rektumkarzinoms sind nach deutscher S3-Leitlinie folgende drei Operationen als onkologisch gleichwertig anzusehen: die (tiefe) anteriore Rektumresektion, die abdomino-perineale Rektumexstirpation und die intersphinktäre Rektumresektion. Für alle drei OP-Verfahren gelten die gleichen onkologischen Grundprinzipien:

- bei Karzinomen des mittleren und unteren Rektumdrittels ist eine TME bis zum Beckenboden indiziert, bei Karzinomen des oberen Rektumdrittels eine PME

- der anzustrebende aborale Sicherheitsabstand richtet sich nach Lokalisation und Differenzierungsgrad des Tumors: bei Tumoren des oberen Rektumdrittels beträgt er $5 \mathrm{~cm}$, bei G1/2-Tumoren des unteren Drittels 1-2 cm, bei G3/4-Tumoren des unteren Drittels $>2 \mathrm{~cm}$; bei neoadjuvant therapierten Patienten kann beim tiefsitzenden Rektumkarzinom auch ein minimaler Sicherheitsabstand von 0,5 cm noch akzeptiert werden, wenn dadurch eine Exstirpation vermieden werden kann

- bei am Tumor adhärenten Strukturen soll stets eine en-bloc-Resektion erfolgen

- die autonomen pelvinen Nervenplexus sollten-sofern onkologisch vertretbar-stets geschont werden

\subsubsection{Abdomino-perineale Rektumexstirpation (APR)}

Wenn aufgrund von Infiltration des Sphinkters oder Analkanals kein Kontinenzerhalt möglich ist, gilt die extralevatorische „zylindrische“ abdomino-perineale Rektumexstirpation nach Holm als der Goldstandard. Allerdings liegen hierzu keine randomisierten Studien vor. Ziel der extralevatorischen APR nach Holm ist es, ein Coning 
bzw. eine Taillenbildung durch Kombination der TME mit einer erweiterten Resektion auf Levatorenhöhe zu verhindern, um das Risiko eines Lokalrezidivs in diesem kritischen Abschnitt zu minimieren. Der abdominelle Part der Operation kann laparoskopisch oder offen erfolgen. Der perineale Anteil wird offen durchgeführt mit in Steinschnitt- oder Götzelagerung befindlichem Patienten. Die Operation beginnt abdominell mit dem Herauslösen des Colon descendens und Sigmas aus den sekundär retroperitonealen Verwachsungen. Ein hohes Absetzen der Vena mesenterica inferior (VMI) wie bei der TAR ist bei der APR nicht notwendig. Die Arteria mesenterica inferior (AMI) wird freipräpariert und 1-2 cm nach ihrem Abgang aus der Aorta abgesetzt; alternativ dazu kann in diesem Abschnitt auch lymphadenektomiert und die AMI erst nach dem Abgang der A. colica sinistra abgesetzt werden. Oralwärts muss die Resektionsgrenze so gewählt werden, dass die Länge zur spannungsfreien Ausleitung eines gut perfundierten Descendostomas ausreicht. Die TME erfolgt wie unter 26.4.2 beschrieben. Nach distal erfolgt die Präparation bis zu den Levatorenschenkeln, die dann als Teil des Präparates mitreseziert werden.

Die perineale Rekonstruktion nach abdomino-perinealer Rektumexstirpation kann auf verschiedene Arten und Weisen erfolgen: ist der Defekt nur mäßig groß und beispielweise bei kräftigem Omentum eine das Becken ausfüllende Omentumplastik verfügbar, so kann der perineale Hautdefekt mittels Naht primär verschlossen werden. Bei sehr ausgedehnten Defekten hingegen ist die Deckung durch einen myokutanen Lappen sinnvoll. Infrage kommen hier vor allem der VRAM-Lappen oder ein uni-/bilateraler Schwenklappen des Musculus glutaeus maximus. Auch Rekonstruktionen unter Verwendung von Kunststoff- oder Bionetzen zur Prävention perinealer Hernien sind Optionen; allerdings muss hier zumindest eine kutane Deckung des Netzes spannungsarm möglich sein. Insbesondere nach neoadjuvanter Therapie oder nach extralevatorischer Rektumexstirpation treten perineal häufig Wundkomplikationen und chronische Wundheilungsstörungen auf, die nicht selten chronische perineale Schmerzen und bleibende Fisteln für den betroffenen Patienten nach sich ziehen. Daher sollte vor allem bei voraussichtlich großem perinealem Defekt oder Vorbestrahlung präoperativ eine plastische Defektdeckung mittels myokutanem Lappen in Betracht gezogen und vorbereitet werden. Auch nach deutscher S3-Leitlinie kann nach neoadjuvanter Therapie bzw. bei großem perinealem Defekt die primäre plastische Rekonstruktion durch einen myokutanen Lappen erfolgen. Der VRAM wird in der Regel von rechts gehoben, da das endständige Kolostoma nach Rektumexstirpation im linken Mittelbauch ausgeleitet wird. Der Muskelbauch wird dabei aus der Rektusscheide herauspräpariert und nach kranialer Absetzung zur Defektdeckung im kleinen Becken positioniert. Dies kann bei Bedarf unter Mitnahme einer Hautinsel zur kutanen Deckung perineal erfolgen. Da der Musculus rectus abdominis von den Vasa epigastrica inferiora versorgt wird, empfiehlt es sich insbesondere bei abdominell voroperierten Patienten, die Durchgängigkeit der rechtsseitigen epigastrischen Gefäße sonographisch vor geplanter VRAM-Lappenplastik zu verifizieren. Die zur Verfügung stehende Evidenz zum VRAM-Lappen besteht überwiegend aus 
Kohortenstudien und Fallserien. Immerhin erschien 2014 auch eine RCT - wenn auch mit kleiner Fallzahl $(n=60)$-, die die Ergebnisse nach perinealer Rekonstruktion mit Primärnaht versus VRAM-Lappen im Rahmen einer Rektumexstirpation bei tiefsitzendem Rektumkarzinom verglich. Es ergaben sich signifikant mehr perineale Wundkomplikationen nach primärer Naht (5/30 im VRAM-Arm [17\%], 14/30 im Primärnaht Arm [46\%]). Eine aktuelle systematische Übersichtsarbeit schloss neben der zitierten RCT noch 9 nicht-randomisierte Studien ein, von denen 7 den VRAM-Lappen und zwei eine Gracilisplastik mit der Primärnaht verglichen. Die Metaanalyse der Studiendaten ergab, dass perineale Wundkomplikationen nach Primärnaht mehr als doppelt so häufig auftraten wie nach Lappendeckung.

\subsubsection{Rekonstruktionstechniken nach tiefer Rektumresektion}

Die Wiederherstellung der Darmkontinuität erfolgt nach der onkologischen, sphinktererhaltenden Resektion durch eine Seit-zu-End Anastomose, einen Kolon-J-Pouch, eine transverse Koloplastie oder eine gerade koloanale Anastomose, wobei die beiden erstgenannten Rekonstruktionsoptionen wegen ihrer besseren funktionellen Ergebnisse bevorzugt werden sollten. Auch die deutsche S3-Leitlinie schlussfolgert, dass auf Basis der vorhandenen Evidenz eine gerade koloanale Anastomose vermieden und stattdessen ein Kolon-J-Pouch (mit einer maximalen Schenkellänge von $6 \mathrm{~cm}$ ) oder alternativ eine Seit-zu-End Anastomose, die dem Kolon-J-Pouch vermutlich funktionell äquivalent ist, angelegt werden sollten. Um den Stellenwert der Seit-zuEnd Anastomose zu klären und ihre Gleichwertigkeit hinsichtlich der funktionellen Ergebnisse im Vergleich zum Kolon-J-Pouch zu belegen, bedarf es weiterer Fallzahlstarker RCTs.

\subsubsection{Minimal-invasive Operationsverfahren}

\section{Laparoskopische Operation}

Die onkologische Gleichwertigkeit der Laparoskopie gegenüber dem offenen Vorgehen im Kontext der Rektumkarzinomchirurgie konnte mittlerweile durch mehrere randomisiert kontrollierte Studien sowie Metaanalysen von RCTs belegt werden. Auch die deutsche Leitlinie betrachtet daher die laparoskopische Vorgehensweise (siehe Abb.27.3) bei entsprechender Expertise und Erfahrung des Operateurs als onkologisch gleichwertige Alternative zur offenen Chirurgie. Zumindest partiell widersprüchliche Ergebnisse erbrachte eine 2017 publizierte Metaanalyse 14 randomisiert-kontrollierter Studien mit 4034 Patienten, die das pathoanatomische Outcome nach laparoskopischer versus offener Rektumresektion verglich. Primäre Endpunkte waren die Rate an positiven CRM $(<1 \mathrm{~mm})$ sowie die TME-Qualität. Die Metaanalyse der 5 RCTs, die die TME-Qualität untersuchten, fand eine signifikant bessere TME- 
Qualität, definiert als Intaktheit der mesorektalen Faszie, nach offener Resektion: bei 13,2\% der Patienten nach laparoskopischer versus 10,4\% der Patienten nach offener Rektumresektion war die Mesorektum-Exzision inkomplett (RR 1,31; KI: 1,05-1,64; $\mathrm{p}=0,02$ ). Alle anderen untersuchten pathoanatomischen Surrogatparameter der onkologischen Resektionsqualität unterschieden sich hingegen nicht. Inwieweit sich die schlechtere TME-Qualität auch auf das onkologische Outcome (Lokalrezidivrate, Überleben) auswirkt, müssen die Langzeitergebnisse der eingeschlossenen Studien in Zukunft noch klären.

\section{Roboter-assistierte Operation}

Zunehmend kommt neben der konventionellen Laparoskopie auch das Roboter-assistierte laparoskopische Vorgehen im Bereich der onkologischen Rektumchirurgie zur Anwendung. Die propagierten Vorteile des Roboters sind die hohe Ergonomie, die freie Angulation mit bis zu 7 Freiheitsgraden, die dreidimensionale Visualisierung des Situs und die Aufhebung des Tremors des Operateurs. Nachteile des Roboter-assistierten Vorgehens stellen die hohen Kosten, lange OP-Dauern und das fehlende taktile Feedback in Bezug auf das zu präparierende Gewebe dar. Hochwertige wissenschaftliche Evidenz, die eine Überlegenheit des Roboter-assistierten Vorgehens gegenüber dem konventionell laparoskopischen belegt, fehlt bis heute. Die Machbarkeit und Sicherheit der robotischen Rektumkarzinomchirurgie konnten dagegen in multiplen, nicht randomisierten Studien gezeigt werden. In der 2017 publizierten multizentrischen ROLARR RCT, die an 471 randomisierten Patienten die konventionell laparoskopische mit der Roboter-assistierten onkologischen Rektumresektion verglich, ergab sich weder ein signifikanter Unterschied hinsichtlich der Rate an Konversionen auf einen offenen Zugang, dem primären Endpunkt der Studie, noch hinsichtlich der sekundären Endpunkte wie Morbidität und Mortalität, onkologische Surrogatparameter, Lebensqualität, Blasen- und Sexualfunktion. Deshalb schlussfolgert die deutsche Leitlinie auch, dass die Roboter-assistierte Vorgehensweise weiter als „experimentell anzusehen“ ist, was in noch größerem Ausmaß auf NOTES (natural orifice transluminal endoscopic surgery), transanale Methoden oder sonstige innovative Techniken zutreffe.

Eine weitere aktuelle koreanische Phase II-RCT mit 139 Rektumkarzinom-Patienten (Einschlusskriterium: Tumorhöhe $\leq 9 \mathrm{~cm}$ ab ano; Ausschlusskriterien: T4-Kategorie, Fernmetastasen) verglich ebenfalls die konventionell laparoskopische mit der robotisch-assistierten Vorgehensweise. Die TME-Qualität, primärer Endpunkt der Studie, unterschied sich nicht zwischen den beiden Armen. Der Roboter-assistiert operierte Arm wies signifikant längere OP-Dauern und eine höhere Anzahl resezierter Lymphknoten auf. Auch die Rate an erreichter Mindestanzahl von $\geq 12$ Lymphknoten war höher in diesem Arm. Alle anderen pathohistologischen Surrogatparameter des onkologischen Outcomes unterschieden sich nicht signifikant, ebenso wenig wie die 
Lebensqualität. Allerdings gaben die robotisch-operierten Patienten eine signifikant bessere Sexualfunktion ein Jahr nach der Operation an.

\subsection{Perioperative Komplikationen und Komplikationsmanagement}

Unter den intraoperativen Komplikationen zählen Verletzungen von Harnleiter (vor allem des linken), Blase und Vagina oder anderen Nachbarorganen des Rektums sowie Blutungskomplikationen zu den relevantesten. Verletzungen der Harnblase lassen sich meist problemlos mittels Naht versorgen, ggf. mit Unterstützung eines urologischen Kollegen. Harnleiterverletzungen sollten umgehend behandelt werden. Die zu wählende Technik hängt von Ausmaß und Höhe der Läsion ab: während kleine Läsionen mittels Doppel-J-Katheter oder perkutaner Nephrostomie versorgt werden können, kommen beispielsweise nach Verletzung des oberen und mittleren Ureters Ureteroureterostomie und Ureterotransversostomie infrage. Bei Verletzung des unteren Ureters können eine Ureterreimplantation, Psoas-bladder-hitch-Plastik oder Zystoplastik indiziert sein, bei einer den proximalen oder kompletten Ureter betreffenden Läsion sind das Ileuminterponat, aber als ultima ratio auch die Autotransplantation und Nephrektomie zur Verfügung stehende Optionen.

Verletzungen der Vagina(hinterwand) können meist mittels Naht rekonstruiert werden.

$\mathrm{Zu}$ Blutungskomplikationen kann es beispielsweise bei Mobilisation der linken Flexur im Bereich der Milz oder bei Verletzung der Iliakalgefäße kommen. Läsionen an den Iliakalgefäßen sollten mittels monofilem, nicht resorbierbarem Nahtmaterial versorgt werden. Zudem kann es bei nicht schichtgerechter Präparation im kleinen Becken zu schwer kontrollierbaren Blutungen aus dem präsakralen venösen Plexus kommen, welche am ehesten mittels Kompression erfolgreich gestillt werden. Falls unter Kompression keine suffiziente Blutstillung zu erzielen ist, sollte das „Packing“ des Beckens mit Bauchtüchern erwogen werden. Nach Gerinnungsoptimierung und Stabilisierung des Patienten kann die Operation in einer zweiten Sitzung komplettiert werden.

$\mathrm{Zu}$ den relevantesten postoperativen Komplikationen nach Rektumresektion gehören Nachblutungen, Wundinfekte, Anastomoseninsuffizienz, Blasenentleerungsstörungen und Stomakomplikationen. Darunter ist die Anastomoseninsuffizienz nach wie vor eine der gefürchtetsten Komplikationen. Um vor allem die aus der Anastomoseninsuffizienz resultierende Morbidität und Mortalität zu senken, empfiehlt die deutsche Leitlinie evidenzbasiert, im Rahmen einer tiefen anterioren Rektumresektion mit tiefer Anastomose die Anlage eines Deviationsstomas. Wie eine systematische Übersichtsarbeit nach Cochrane-Methodik belegen konnte, lassen sich durch diese protektive Maßnahme die Morbidität im Falle einer Anastomoseninsuffizienz und die daraus häufig resultierende Rate an Notfall-Relaparotomien signifikant reduzieren. 
Sofern bereits im Rahmen der Primäroperation ein protektives Ileostoma angelegt wurde, kann die Anastomose in der Regel erhalten und ggf. mittels Endo-Vac-Therapie zum Ausheilen gebracht werden. Aus der Anastomoseninsuffizienz resultierende Beckenabszesse können beispielsweise CT-gesteuert drainiert werden, sofern bei der Primäroperation keine Drainage im Bereich des kleinen Beckens platziert wurde oder diese den Abszess nicht ausreichend drainiert. Erscheint aufgrund der Größe des Defektes ein Anastomosenerhalt nicht möglich oder gerät der Patient in eine fulminant septische Situation, so muss die Indikation zur Relaparotomie und Lavage, ggf. mit Auflösung der Anastomose, großzügig gestellt werden. Im Falle einer segmentären oder weitergehenden Nekrose des transponierten Kolons als Ursache einer Anastomoseninsuffizienz muss die Anastomose aufgehoben und der nekrotische Darmanteil reseziert werden.

Störungen der Sexualfunktion und der Stuhlentleerung sowie die Ausbildung einer Anastomosenstenose gehören zu den besonders patientenrelevanten Spätkomplikationen, die die Lebensqualität des Patienten maßgeblich beeinflussen. In diesem Kontext zu erwähnen ist das sogenannte „Low Anterior Resection Syndrome“ (LARS), das sich klinisch als Mischbild aus hoher Stuhlfrequenz, fragmentierter Entleerung und Inkontinenz präsentiert. Mangels einer einheitlichen Definition des Syndroms variieren auch die Angaben in der Literatur bezüglich der LARS-Raten und reichen von $25-50 \%$. Faktoren, die zu den Ursachen von LARS gerechnet werden, sind unter anderem der operativ bedingte Sphinkterschaden und die autonome Denervierung. Therapieoptionen bei LARS umfassen Sakralnerven- oder Tibialis posterior-Stimulation, Beckenbodentraining, Probiotika- oder 5-HT3-Rezeptor-Antagonisten. Allerdings ist die Evidenz hierzu noch limitiert.

Eine Anastomosenstenose, die nach stattgehabter Anastomoseninsuffizienz vermehrt auftritt, kann mittels endoskopischer Ballondilatation, Stents oder auch durch eine chirurgische Nachresektion therapiert werden.

\subsection{Prognose}

Die Prognose hängt von Patienten-, Tumor- und Therapie-assoziierten Faktoren ab, wobei die Radikalität und Qualität der chirurgischen Resektion eine entscheidende Rolle spielen. $\mathrm{Zu}$ den bekannten negativen Prognosefaktoren zählen u. a. extramurale venöse Infiltration (EMVI), CRM-Positivität, inkomplette bzw. schlechte TMEQualität, intraoperative Tumorperforation, Lymphknotenbefall, fortgeschrittene T-Kategorie, Fernmetastasierung und BRAF-Mutation. Ein positiver Prognostikator und Prädiktor hingegen ist beispielsweise die hochgradige Mikrosatelliteninstabilität (MSI). Laut deutscher Leitlinie gilt die Anzahl resezierter und untersuchter Lymphknoten ebenfalls bei den UICC Stadien II und III als positiver Prognosefaktor, da sie als Surrogatparameter der chirurgischen Qualität zu werten ist. 
Aktuelle populations-basierte deutsche Krebsregisterdaten zeigen nicht nur einen prognostischen Vorteil bei Behandlung in einem zertifizierten Darmkrebszentrum, sondern belegen auch die Sicherheit der laparoskopischen Rektumkarzinom-Chirurgie. Nach potentiell kurativer Resektion fand sich ein 5-Jahres-Gesamtüberleben von $69 \%$ nach offener und $80 \%$ nach laparoskopischer Resektion. Stadienspezifisch liegt das 5-Jahres-Überleben ohne perioperative Todesfälle bei $83 \%$ bzw. $91 \%$ im Stadium I, $71 \%$ bzw. 79\% im Stadium II und $68 \%$ bzw. $80 \%$ im Stadium III (jeweils nach offener bzw. laparoskopischer Resektion, ohne perioperative Todesfälle). Auch wenn es sich hierbei nicht um randomisierte Studien, sondern Registerdaten mit einem relativ höheren Anteil von leitliniengerechten multimodalen Therapien und höherem Anteil von Zentrums-Behandlungen in der laparoskopisch resezierten Gruppe handelt, zeigten multivariate Analysen in dieser Studie, dass eine laparoskopische Resektion per se prognostisch sicher nicht nachteilig ist und dass es heute sogar in der Fläche und nicht nur an speziellen Zentren möglich sein sollte, bis zu drei von vier Patienten mit primär nicht-metastasiertem Rektumkarzinom ein Langzeitüberleben zu ermöglichen.

\section{Weiterführende Literatur}

Al-Sukhni E, Milot L, Fruitman M, et al. Diagnostic accuracy of MRI for assessment of T category, lymph node metastases, and circumferential resection margin involvement in patients with rectal cancer: a systematic review and meta-analysis. Annals of surgical oncology. 2012;19:2212-2223.

Brown CJ, Fenech DS, McLeod RS. Reconstructive techniques after rectal resection for rectal cancer. Cochrane Database Syst Rev. 2008: CD006040.

Devulapalli C, Jia Wei AT, DiBiagio JR, et al. Primary versus Flap Closure of Perineal Defects following Oncologic Resection: A Systematic Review and Meta-Analysis. Plastic and reconstructive surgery. 2016;137:1602-1613.

Fazio VW, Zutshi M, Remzi FH, et al. A randomized multicenter trial to compare long-term functional outcome, quality of life, and complications of surgical procedures for low rectal cancers. Ann Surg. 2007;246:481-488; discussion 8-90.

Holmer C, Benz SR, Fichtner-Feigl S, et al. Transanale totale mesorektale Exzision (taTME) - eine kritische Standortbestimmung. Der Chirurg. 2019 (zur Publikation akzeptiert).

Jayne DG, Thorpe HC, Copeland J, et al. Five-year follow-up of the Medical Research Council CLASICC trial of laparoscopically assisted versus open surgery for colorectal cancer. Br J Surg. 2010;97:1638-1645.

Jayne D, Pigazzi A, Marshall H, et al. Effect of Robotic-Assisted vs Conventional Laparoscopic Surgery on Risk of Conversion to Open Laparotomy Among Patients Undergoing Resection for Rectal Cancer: The ROLARR Randomized Clinical Trial. JAMA. 2017;318:1569-80.

Kapiteijn E, Marijnen CA, Nagtegaal ID, et al. Preoperative radiotherapy combined with total mesorectal excision for resectable rectal cancer. N Engl J Med. 2001;345:638-646.

Kim MJ, Park SC, Park JW, et al. Robot-assisted Versus Laparoscopic Surgery for Rectal Cancer: A Phase II Open Label Prospective Randomized Controlled Trial. Ann Surg. 2018;267:243-251. 
Kong JC, Guerra GR, Warrier SK, Ramsay RG, Heriot AG. Outcome and Salvage Surgery Following „Watch and Wait" for Rectal Cancer after Neoadjuvant Therapy: A Systematic Review. Dis Colon Rectum. 2017;60:335-345.

Kreis ME, Straßburg, J. (Hrsg.). Moderne Chirurgie des Rektumkarzinoms. Springer, 2015.

Kuhry E, Schwenk WF, Gaupset R, Romild U, Bonjer HJ. Long-term results of laparoscopic colorectal cancer resection. Cochrane Database Syst Rev 2008: CD003432.

Leitlinienprogramm Onkologie (Deutsche Krebsgesellschaft DK, AWMF). S3 Leitlinie Kolorektales Karzinom, Langversion 2.0, 2017.

Lefevre JH, Mineur L, Kotti S, et al. Effect of Interval (7 or 11 weeks) Between Neoadjuvant Radiochemotherapy and Surgery on Complete Pathologic Response in Rectal Cancer: A Multicenter, Randomized, Controlled Trial (GRECCAR-6). J Clin Oncol. 2016;34:3773-3780.

Liao C, Gao F, Cao Y, et al. Meta-analysis of the colon J-pouch vs transverse coloplasty pouch after anterior resection for rectal cancer. Colorectal Dis. 2010;12:624-631.

Martinez-Perez A, Carra MC, Brunetti F, de'Angelis N. Pathologic Outcomes of Laparoscopic vs Open Mesorectal Excision for Rectal Cancer: A Systematic Review and Meta-analysis. JAMA Surg. 2017;152:e165665.

Penna M, Hompes R, Arnold S, et al. Transanal Total Mesorectal Excision: International Registry Results of the First 720 Cases. Ann Surg. 2017;266:111-117.

Petrelli F, Sgroi G, Sarti E, Barni S. Increasing the Interval Between Neoadjuvant Chemoradiotherapy and Surgery in Rectal Cancer: A Meta-analysis of Published Studies. Ann Surg. 2016;263:458-464.

Pettersson D, Lorinc E, Holm T, et al. Tumour regression in the randomized Stockholm III Trial of radiotherapy regimens for rectal cancer. Br J Surg. 2015;102:972-978; discussion 8.

Puli SR, Reddy JB, Bechtold ML, et al. Accuracy of endoscopic ultrasound to diagnose nodal invasion by rectal cancers: a meta-analysis and systematic review. Annals of surgical oncology. 2009;16:1255-1265.

Sauer R, Becker H, Hohenberger W, et al. Preoperative versus postoperative chemoradiotherapy for rectal cancer. N Engl J Med. 2004;351:1731-1740.

Sauer R, Liersch T, Merkel S, et al. Preoperative versus postoperative chemoradiotherapy for locally advanced rectal cancer: results of the German CAO/ARO/AIO-94 randomized phase III trial after a median follow-up of 11 years. J Clin Oncol. 2012;30:1926-1933.

Tanaka A, Sadahiro S, Suzuki T, Okada K, Saito G. Comparisons of Rigid Proctoscopy, Flexible Colonoscopy, and Digital Rectal Examination for Determining the Localization of Rectal Cancers. Dis Colon Rectum. 2018;61:202-206.

Van den Broek CB, Vermeer TA, Bastiaannet E, et al. Impact of the interval between short-course radiotherapy and surgery on outcomes of rectal cancer patients. Eur J Cancer. 2013;49:3131-3139.

van Gijn W, Marijnen CA, Nagtegaal ID, et al. Preoperative radiotherapy combined with total mesorectal excision for resectable rectal cancer: 12-year follow-up of the multicentre, randomised controlled TME trial. Lancet Oncol. 2011;12:575-582.

Wang XJ, Zheng ZR, Chi P, et al. Effect of Interval between Neoadjuvant Chemoradiotherapy and Surgery on Oncological Outcome for Rectal Cancer: A Systematic Review and Meta-Analysis. Gastroenterol Res Pract. 2016;2016:6756859. 
